\title{
La fabrique des catastrophes « naturelles »
}

\author{
Alexandre Magnan ${ }^{1}$, Virginie Duvat ${ }^{2}$ \\ 1 Géographe, IDDRI, 75337 Paris Cedex 07, France \\ 2 Géographe, Université de la Rochelle, UMR 7266 LIENSs, 17000 La Rochelle, France
}

\begin{abstract}
On observe depuis quelques décennies une aggravation des impacts humains et économiques des phénomènes naturels. On attend par ailleurs un renforcement des événements dits extrêmes dans le contexte actuel du changement climatique. À l'interface entre sociétés et nature, les auteurs de cet article s'interrogent sur ce que l'impact croissant des phénomènes naturels dit de nos sociétés, tel un miroir où nous reconnaissons les liens distendus entre l'humanité et son environnement naturel, la perte de notre mémoire collective face aux réalités des risques environnementaux, et finalement la persistance de notre vulnérabilité en dépit de notre technologie. Un texte qui invite à nous engager dès maintenant sur la voie pragmatique de l'adaptation aux changements en cours, rappelant que cela n'est pas hors de notre portée.
\end{abstract}

La Rédaction

\section{Mots-clés :}

catastrophes naturelles; risques littoraux ; vulnérabilité adaptation au changement climatique; tempêtes ; cyclones; tsunamis

\section{Keywords:}

natural disaster; coastal risks; vulnerability; adaptation to climate change; storms; tropical cyclones; tsunamis

Résumé - Ce texte s'attache à mettre en évidence les causes profondes de la vulnérabilité des sociétés aux aléas naturels en exposant les facteurs et les processus qui transforment un simple phénomène naturel en catastrophe. À partir de trois études de cas (le tsunami de 2004 aux Maldives, Katrina en 2005 aux États-Unis, Xynthia en 2010 en France), les auteurs montrent le rôle de quatre grands facteurs, identifiés comme étant universels, dans la fabrique des catastrophes «naturelles » : la conquête d'espaces exposés aux aléas météomarins, la dégradation des zones tampons naturelles, le " mythe du développement sûr » et la perte des liens que les sociétés entretiennent avec leur environnement.

\begin{abstract}
The making of "natural" disasters. To contribute an explanation for the increasing number of natural disasters, occurring especially in coastal areas, this paper highlights their root causes, i.e. the drivers and processes that make natural events, such as storms or tsunamis, turn into disasters due to the adverse impacts they have on human societies. Based on case studies, the authors identify four key and universal factors driving the generation of natural disasters throughout the world: the settlement of areas that are highly exposed to natural hazards, the degradation of natural buffers, the "myth of safe development" and the loss of environmental knowledge. The three case studies presented here illustrate the processes by which these drivers produce a "risk system" that will make a natural hazard generate a natural disaster: Tropical Cyclone Katrina in the United States (2005), the Xynthia Storm in France (2010) and the Sumatra Tsunami in the Maldives (2004). Based on the lessons learnt from these case studies, this paper outlines key principles for action, arguing in particular that tackling the root causes of current vulnerability is therefore a pragmatic pathway to implement adaptation to climate change.
\end{abstract}

\section{Introduction}

Les côtes françaises ont été sérieusement affectées par les tempêtes qui ont sévi tout au long de l'hiver 2013-2014. Les images de dunes et de plages érodées, d'habitations menacées d'être emportées par la mer, de vagues s'écrasant sur les digues des ports de plaisance, etc., restent dans notre mémoire collective. Toutes ces images ne sont pas sans en rappeler d'autres, en France (la tempête Xynthia en février 2010 sur les côtes de Charente-Maritime et de Vendée, le cyclone Bejisa sur l'île de La Réunion en janvier 2014) ou à l'étranger (par

Auteur correspondant : A. Magnan, alexandre.magnan@iddri.org

Virginie Duvat est membre du Groupe d'experts intergouvernemental sur l'évolution du climat (GIEC). 
exemple, le cyclone Haruna à Madagascar en février 2013 et le typhon Haiyan aux Philippines en novembre 2013). Ces événements ont eu des impacts importants, bien que très variables selon les territoires touchés et $l^{\prime}$ intensité des aléas ${ }^{1}$ déclencheurs. Plus largement, on assiste dans le monde depuis plusieurs décennies à une multiplication des impacts tant humains (personnes affectées) qu'économiques des phénomènes naturels, dont près des deux tiers sont d'origine climatique (inondations, tempêtes, températures extrêmes, sécheresses) (voir, par exemple, Dworkin, 1974 ; Guha-Sapir et al., 2004 ; WEF, 2014). Dans un contexte de changement climatique, un tel constat amène à se poser la question essentielle de la part de la nature et de la part de l'homme dans l'augmentation du nombre de catastrophes ${ }^{2}$.

La situation des littoraux est exemplaire pour identifier les facteurs anthropiques qui contribuent à la production des catastrophes. Elle illustre comment, de façon insidieuse et sur un temps long, les sociétés "fabriquent » les catastrophes naturelles. Privilégier un angle d'entrée « humain » ne doit pas être interprété ici comme une volonté de minimiser le rôle des facteurs naturels, mais davantage comme un souci de mettre à jour la responsabilité conjointe des sociétés et de leurs modes de développement dans la fréquence et l'ampleur de ces catastrophes.

Il n'est donc aucunement paradoxal ici d'utiliser le terme "naturelles ». Si de nombreuses voix s'élèvent aujourd'hui, principalement en dehors de la communauté scientifique et au travers des médias, contre ce qualificatif, arguant que seules les sociétés sont responsables, il nous semble au contraire primordial de le maintenir, et ce pour au moins deux raisons. La première est que ce sont bien des aléas naturels qui déclenchent les catastrophes dont il est question ici, c'est-à-dire des événements dont $l^{\prime}$ homme n'est pas à l'origine (les cyclones en sont un bon exemple). À ce titre, les catastrophes «naturelles » se distinguent de celles déclenchées par des aléas d'origine industrielle ou économique, par exemple. Une seconde raison, plus fondamentale, est que ce terme a pour mérite de rappeler que les sociétés

1 Les aléas naturels sont les phénomènes naturels qui peuvent se produire en un lieu donné et qui constituent une source de danger pour l'homme. Ils se caractérisent par une probabilité d'occurrence, c'est-à-dire une chance de survenir. On ne peut en général pas déterminer à l'avance quand ils vont se produire.

2 Dans ce texte, le terme de « catastrophe » renverra au processus d'enchaînement d'impacts qui est déclenché, dans un territoire donné, par la survenue d'un aléa naturel. Si l'aléa se transforme en catastrophe, c'est parce que le territoire affecté peut être caractérisé comme un « système du risque » (voir note de bas de page suivante). humaines ne sont pas à l'abri des forces de la nature et qu'elles conservent une forme de fragilité par rapport aux aléas naturels (Cannon, 1994; Wisner et al., 2004). Or, il nous paraît capital, en particulier dans un contexte de changement climatique, de conserver la conscience de cet état de fait.

Une fois ces éléments généraux de compréhension énoncés, deux questions se posent auxquelles ce texte entend répondre : comment expliquer que l'on passe d'un aléa naturel à une catastrophe, qui prend toute son ampleur sur un territoire donné par ses conséquences humaines, matérielles, économiques, sociales, etc. ? À partir de là, qu'est-ce qui explique l'augmentation du nombre de catastrophes naturelles à l'échelle de la planète?

La première partie de ce texte pose le cadre de la réflexion au travers $d^{\prime}$ une synthèse des grands facteurs anthropiques qui, d'un cas à un autre, montrent la mise en place progressive d'un "système du risque ${ }^{3}$ ", lequel va expliquer pourquoi un aléa spécifique va, à un moment donné, générer une catastrophe. La deuxième partie illustre ce propos au travers de trois exemples concrets de catastrophes récentes: Katrina aux Etats-Unis en 2005, le tsunami de décembre 2004 aux Maldives, et la tempête Xynthia en 2010 en France. La dernière partie, enfin, fait état des principaux enseignements.

\section{Les facteurs anthropiques à l'origine des catastrophes naturelles}

Ce texte s'appuie sur des études de cas développées dans notre ouvrage ${ }^{4}$ paru en 2014 , portant sur des catastrophes récentes survenues dans sept régions du monde (Bangladesh, Caraïbe, États-Unis, France, Japon, Maldives, îles de l'océan Pacifique) et mettant en scène différents types d'aléas (tempêtes, cyclones et tsunamis). Pour chacune de ces études de cas, il s'est agi d'analyser les processus qui expliquent, aux côtés de la survenue d'un aléa d'intensité forte ou extrême, le déclenchement d'une catastrophe. L'un des enjeux a donc consisté à identifier les racines profondes de chacune de ces catastrophes, en couvrant à la fois des événements qui ont frappé des pays en développement et

\footnotetext{
3 Ce texte entend par «système du risque » le modèle qui explique comment différents facteurs aux dynamiques propres mettent progressivement en place, par leurs interactions plus ou moins directes et au fil du temps, les conditions de propagation, par effet domino, des impacts d'un événement spécifique (ici, un aléa naturel) à de multiples composantes du système affecté (ici, un territoire littoral). Ainsi, le "système du risque" explique la mise en place du risque qui, lorsque survient l'aléa, devient réalité au travers d'une catastrophe.
}

4 Des catastrophes... «naturelles »? (Duvat et Magnan, 2014). 
des pays développés afin de mettre en évidence les fondements communs de la vulnérabilité des sociétés aux aléas naturels.

$\mathrm{Au}$ terme de ces études de cas, quatre facteurs anthropiques principaux expliquant la survenue de catastrophes sur le littoral et, par conséquent, l'augmentation de leur nombre à l'échelle de la planète, ont pu être identifiés.

\section{Facteur 1 : la conquête d'espaces exposés aux aléas météomarins}

En premier lieu, l'homme occupe toujours davantage d'espace à l'échelle de la planète et les littoraux constituent depuis plusieurs décennies, et encore aujourd'hui, des lieux privilégiés d'extension et de densification du peuplement. Ce facteur entraîne logiquement l'occupation croissante d'espaces qui sont naturellement soumis à l'influence d'aléas naturels. On en trouve des exemples partout: sur l'atoll de Tarawa dans l'archipel des Kiribati (Pacifique central), où la flèche sableuse instable d'Eita-Bangantebure connaît une forte croissance démographique du fait de populations venues des atolls ruraux vers l'atoll capitale (Duvat et al., 2013) ; la baie de Saint-Paul sur la côte ouest de l'île de La Réunion, qui a connu une densification importante de population entre le XVIII ${ }^{\mathrm{e}}$ siècle et aujourd'hui ; ou encore des zones de cuvettes, telles que celle de La Faute-sur-Mer en Vendée, qui a été fortement affectée par la tempête Xynthia en février 2010 (Chauveau et al., 2011 ; Vinet et al., 2012).

Ainsi les sociétés humaines s'exposent d'elles-mêmes de plus en plus à des phénomènes naturels qui, en retour, sont de plus en plus susceptibles de produire des effets catastrophiques.

\section{Facteur 2 : la dégradation des zones tampons naturelles}

En deuxième lieu, les sociétés humaines, en modifiant les milieux naturels dans lesquels elles s'implantent, tendent à détruire les fonctions protectrices et la résilience de certains écosystèmes comme les dunes, les récifs coralliens, les mangroves et les forêts littorales en général. Or, il est établi que ces écosystèmes jouent un rôle tampon face aux aléas naturels en atténuant leurs impacts, parce qu'ils absorbent l'énergie des vagues ou la force des vents, par exemple. De nombreux types $\mathrm{d}$ 'interventions humaines contribuent de fait à la dégradation, voire à la destruction de ces zones tampons : l'aménagement de ports et l'ouverture de chenaux de navigation dans les platiers récifaux (Cazes-Duvat, 2007), les travaux de déforestation qui affectent les cordons littoraux et les vasières à mangrove, l'extraction de matériaux et la création de terre-pleins, la construction d'ouvrages de défense déstabilisateurs (Duvat et al., 2013), etc.

\section{Facteur 3 : le mythe de la « sûreté »}

En troisième lieu, il est désormais reconnu que les sociétés humaines, particulièrement dans les pays développés, se croient fréquemment, de manière consciente ou non, relativement à l'abri des forces de la nature, et donc des événements extrêmes générateurs de catastrophes. Ce facteur s'explique en grande partie par la prégnance d'une culture « ingénierique » du développement et du rapport à l'espace, aux ressources et aux risques (Paskoff, 2004). Cette culture, qui s'est forgée pendant les Trente Glorieuses dans un contexte de croissance économique soutenant l'essor des capacités financières et techniques des pays développés, engendre très souvent l'illusion d'un développement « sûr » ou " maîtrisé », notamment mis en évidence par Raymond Burby (2006) à la suite du passage de l'ouragan Katrina sur la Nouvelle-Orléans (Louisiane, États-Unis) en 2005. Ce phénomène a opéré de la même manière en amont de la tempête Xynthia en France (Cour des comptes, 2012) ou encore du tremblement de terre et du tsunami de mars 2011 au Japon (Nöggerath et al., 2011; Funabashi et Kitazawa, 2012). Au-delà, il est caractéristique de bien $\mathrm{d}$ 'autres situations à travers le monde.

La figure 1 illustre le fonctionnement de ce mythe du développement "sûr " et la manière dont il explique l'augmentation, sur un temps long, de la vulnérabilité des sociétés face aux aléas naturels. Schématiquement, le développement économique amène à avoir recours à des solutions techniques lourdes (digues, cordons d'enrochement, etc.) considérées comme étant résistantes aux aléas, donc protectrices pour les sociétés littorales. Si ces solutions réduisent, en général dans un premier temps, la vulnérabilité aux aléas naturels, elles engendrent aussi en parallèle et sur un temps plus long l'émergence d'un mythe de la sûreté, autrement dit l'impression de maîtriser les éléments naturels, laquelle mène à son tour à une perte de vigilance face au risque, puis à un affaiblissement des systèmes de prévention et d'anticipation de ce risque. L'ont récemment illustré le manque d'entretien des digues de Charente-Maritime lorsque survint Xynthia ou l'absence de mesures de rétablissement rapide de l'alimentation électrique de la centrale japonaise de Fukushima-Daiichi à la suite du tsunami du 11 mars 2011 (Funabashi, 2012 ; Hasegawa, 2012). Ces éléments se combinent aux facteurs précédemment cités (occupation d'espaces exposés aux aléas naturels et dégradation des zones tampons) pour constituer de puissants facteurs explicatifs de la hausse graduelle de l'exposition des populations, des activités et des biens (facteur 1) et engendrer, à terme, l'accroissement de la vulnérabilité. 


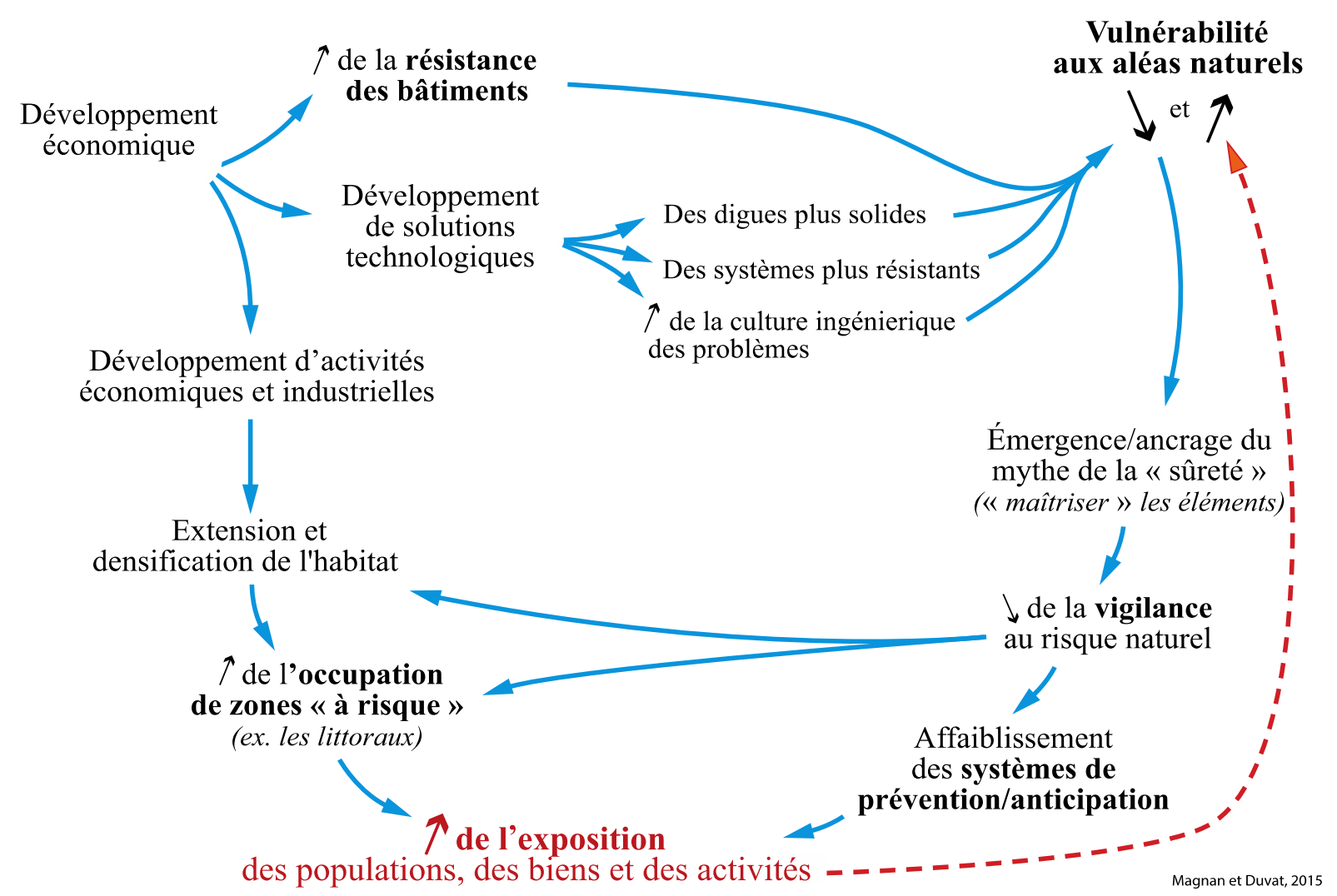

Fig. 1. Du mythe du développement «sûr » à la vulnérabilité de long terme.

\section{Facteur 4 : la perte du lien avec l'environnement}

En quatrième et dernier lieu, s'il est vrai que le processus de développement réduit par certains aspects la vulnérabilité, comme c'est, par exemple, le cas de la construction de maisons en dur dans les régions exposées aux cyclones, il génère en parallèle d'autres formes de vulnérabilité en ce qu'il produit in fine des modes de vie relativement déconnectés des phénomènes naturels. Ce processus conduit en effet à son tour (i) à un déficit de connaissances sur le fonctionnement de l'environnement direct, ce qui nuit à la capacité des populations littorales à repérer les signes avant-coureurs de la catastrophe, (ii) à une perte de la mémoire des événements extrêmes et des catastrophes passées et, finalement, (iii) à un déclin souvent très marqué de la conscience du risque. Ce point d'aboutissement produit ainsi des effets de cercles vicieux, par exemple l'occupation (souvent inconsciente) de zones basses soumises aux aléas, qui engendrent une augmentation de l'exposition des populations et, à terme, de la vulnérabilité.

Dans la réalité, bien entendu, ces quatre facteurs se combinent et s'alimentent réciproquement pour expliquer comment les sociétés façonnent des territoires vulnérables qui basculent, lors de la survenue d'un aléa, dans la catastrophe.

\section{Des exemples de catastrophes révélatrices de la vulnérabilité des sociétés littorales face aux aléas naturels}

Trois exemples de catastrophes récentes illustrent les propos précédents. Ils sont pris dans des pays développés (États-Unis, France) et en développement (Maldives) afin de montrer que les formes de vulnérabilité de ces deux types de sociétés sont en partie partagées.

\section{Exemple 1 : le cyclone Katrina aux États-Unis (2005)}

Prenons en premier lieu le cas de Katrina aux États-Unis en 2005. La ville de la Nouvelle-Orléans a été fondée en 1718 au cœur de la plaine de Louisiane, à la confluence de trois grandes voies navigables (le lac Pontchartrain au nord, le lac Borgne à l'est et le fleuve Mississippi au sud), au cœur de la plus grande zone humide des États-Unis. Elle se situe donc dans une zone de très basse altitude, pour partie au-dessous du niveau de la mer. Elle se situe aussi dans un contexte climatique particulier, celui du golfe du Mexique, qui est propice aux cyclones de forte intensité. La ville a ainsi connu au cours de ses trois siècles d'histoire plus d'une vingtaine de cyclones particulièrement intenses. Les autorités ont donc très tôt érigé des digues de protection pour faire 
face aux inondations régulières dues notamment au débordement des lacs (Kates et al., 2006 ; Mancebo, 2006). $\mathrm{Au}$ fil des cyclones, ces digues ont été rehaussées, et de nouvelles ont été édifiées, notamment vers le nord-est, selon un rythme qui s'est accéléré après le passage du cyclone Betsy en 1965.

Après cet événement, le Congrès américain autorise en effet la construction de vastes digues à la limite du lac Pontchartrain, sans dissimuler que ce projet constitue une opportunité pour conquérir de nouvelles terres et étendre l'agglomération en colonisant les zones humides du Nord-Est (Kates et al., 2006). Le développement de ce réseau a entraîné une extension et une densification de l'urbanisation, sous l'effet du " paradoxe du développement sûr » (Burby, 2006) : la mise en place de nouvelles digues a généralement pour effet pervers de conférer aux zones situées en arrière une image d'espaces " protégés », c'est-à-dire situés à l'abri des aléas météomarins et pouvant par conséquent être aménagés en toute sécurité ; il en résulte généralement que des secteurs naturellement à risque sont de plus en plus habités, ce qui conduit à la catastrophe lorsqu'un aléa survient qui dépasse en intensité la capacité de protection des digues. À la Nouvelle-Orléans, cette urbanisation a également conduit à des problèmes d'évacuation des eaux de pluie, dans des zones naturellement favorables à leur rétention («cuvette» ou plaines côtières de basse altitude). La solution adoptée à la Nouvelle-Orléans pour faire face à ce problème a consisté à quadriller la ville de canaux de drainage, complétés par des systèmes de pompage relativement sophistiqués pour l'époque. Mais ce palliatif n'a pas empêché, au fil du temps, une forte perturbation de l'écoulement naturel des eaux de pluie et une imperméabilisation des sols à grande échelle, deux facteurs qui ont renforcé le risque d'inondation. Parallèlement, le passage du cyclone Katrina s'est produit dans un contexte d'importantes défaillances dans l'entretien des digues et de présence de brèches dans les canaux de drainage. Ces facteurs de fragilisation du système sont très majoritairement imputables à un affaiblissement des moyens financiers et humains de la FEMA (Federal Emergency Management Agency), l'autorité fédérale en charge de la lutte contre les risques naturels. Cet affaiblissement est une conséquence indirecte des attentats terroristes du 11 septembre 2001, à la suite desquels l'administration du président George W. Bush s'est nettement focalisée sur la lutte antiterroriste, au détriment des programmes de prévention des risques naturels (Mancebo, 2006). Lors du passage de Katrina, de fortes pluies combinées à une pression des eaux marines venues du golfe du Mexique ont eu pour conséquence l'engouffrement rapide de l'eau dans les canaux et les digues défaillants, lesquels ont cédé, laissant l'inondation s'emparer de la ville. Au total, ce cyclone a été l'un des plus meurtriers de toute l'histoire des États-Unis (près de 1800 décès dont plus de $80 \%$ dans l'État de Louisiane).

Pourtant, un exercice de simulation avait été mené plusieurs mois auparavant, à partir du cas théorique d'un cyclone d'une intensité analogue à celui de Katrina (Laska, 2004 ; Mancebo, 2006). Toutes les autorités compétentes avaient alors été réunies pendant plusieurs jours pour cet exercice, mais la probabilité d'occurrence d'un tel cyclone n'avait pas été prise au sérieux, de sorte que l'épisode Katrina n'a pu être maîtrisé et s'est transformé en catastrophe.

Ainsi le passage de Katrina a-t-il, par exemple, eu de graves conséquences sociales. Les classes sociales les plus défavorisées se sont trouvées piégées dans la ville, et elles se sont pour partie rendues dans des centres $\mathrm{d}$ 'hébergement où elles ont dû faire face à des situations chaotiques (Cutter, 2006 ; Gemenne, 2010). Ces centres immenses et surpeuplés des jours durant ne comptaient, en effet, aucun stock de nourriture ou d'eau, tandis que les forces de l'ordre se sont rapidement trouvées dépassées par les événements. Des tensions extrêmes sont très vite apparues, accompagnées d'une forte augmentation du taux de criminalité (viols, par exemple). Une autre partie de la population, restée dans les rues, s'est trouvée, quant à elle, bloquée, sans nourriture, sans eau, sans assistance, devant faire face aux nombreux problèmes dus à cette situation d'extrême pénurie. Ces populations ont alors pris d'assaut les magasins, et les forces de l'ordre ont été contraintes de tirer sur les "pilleurs", sans toutefois pouvoir distinguer les actes de survie des actes de pur vandalisme (Mancebo, 2006).

Ces éléments, combinés à d'autres facteurs (Fig. 2), montrent comment s'est «fabriquée » la catastrophe et comment s'est progressivement mis en place un système $\mathrm{du}$ risque, associant plusieurs échelles temporelles (historiques et contemporaines) et plusieurs facteurs explicatifs. L'exemple de Katrina met ainsi en avant quelquesuns des ingrédients explicités plus haut dans ce texte (l'extension de l'habitat dans des zones à risque, le renforcement des effets des aléas par une gestion inappropriée, etc.), pour produire au final une augmentation considérable de l'exposition des populations. En un sens, la catastrophe n'attendait que Katrina.

\section{Exemple 2 : le tsunami du 26 décembre 2004 aux Maldives}

L'exemple des Maldives face au tsunami de 2004 est tout aussi riche d'enseignements, non seulement sur les facteurs de la vulnérabilité des sociétés, mais aussi sur les leviers de leur résilience. En effet, comme on va le voir, au-delà de ce qu'il nous apprend sur les fondements spécifiques de la vulnérabilité des petits États insulaires en développement, le cas maldivien nous livre 


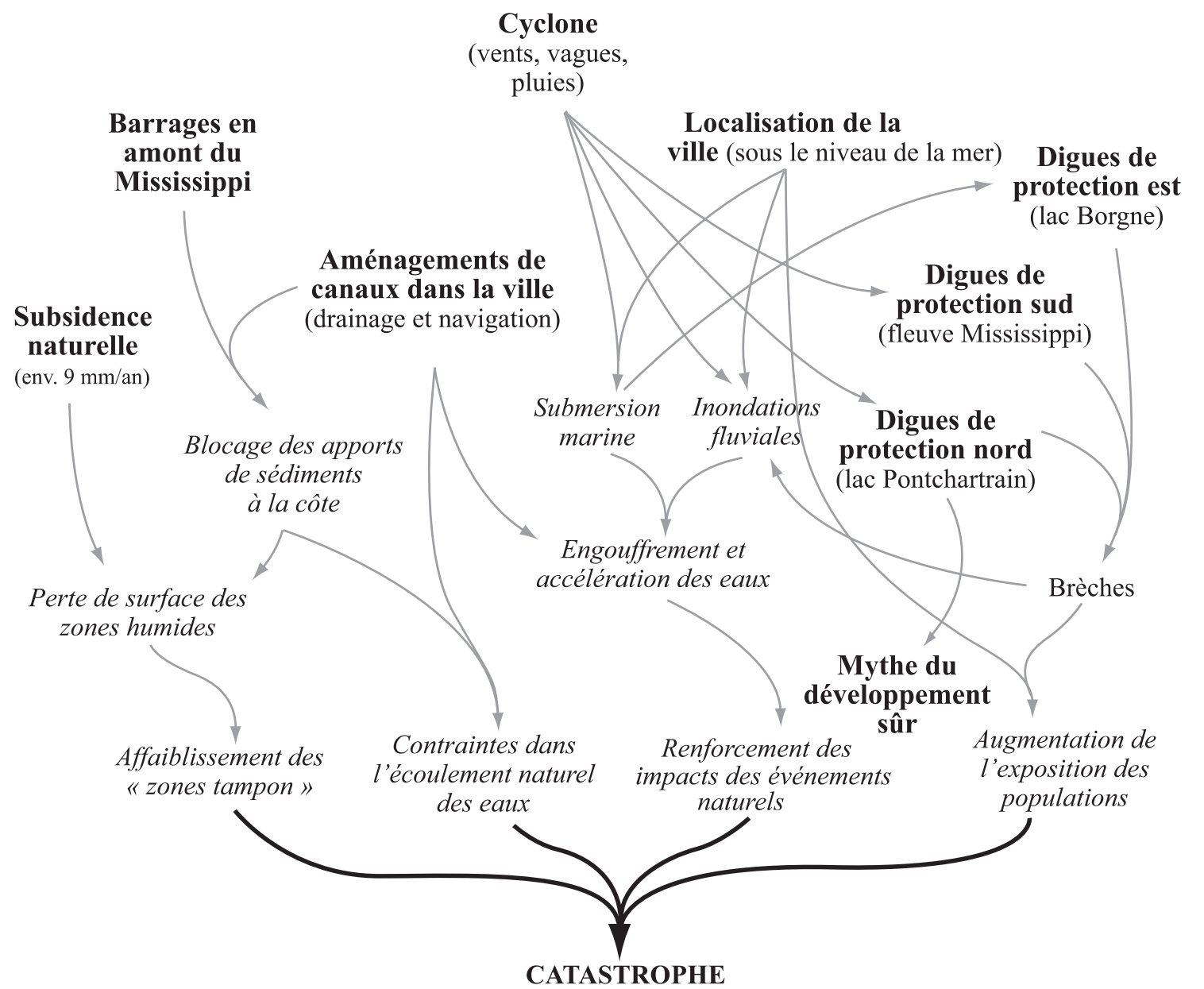

Duvat et Magnan, 2014

Fig. 2. Katrina et le système du risque à la Nouvelle-Orléans.

des éléments fondamentaux sur les facteurs contemporains de l'accroissement de la vulnérabilité des sociétés en général.

Ce petit État insulaire en développement, qui comptait 370000 habitants en 2012 sur une surface émergée de $297 \mathrm{~km}^{2}$, est très exposé aux aléas d'origine marine bien $\mathrm{qu}^{\prime} \mathrm{il}$ se situe en dehors de la zone de formation des cyclones. Cette forte exposition s'explique par deux raisons principales. La première (la plus importante) est sa configuration géomorphologique. En effet, les îles coralliennes qui constituent le territoire maldivien se caractérisent par une faible altitude $(<4 \mathrm{~m})$, une forte mobilité sédimentaire (non seulement saisonnière, commandée par la mousson, mais aussi liée aux aléas météomarins exceptionnels), et une surface moyenne très réduite $\left(0,2 \mathrm{~km}^{2}\right)$. En conséquence, la plupart de ces îles sont sujettes à la submersion sur une vaste partie, voire la totalité, de leur surface. La seconde raison de la forte exposition de ce territoire aux aléas d'origine marine tient aux conséquences de la forte pression démographique qu'il connaît (doublement de la population tous les 25 ans), qui se traduit par des densités de population très élevées (55 000 à 80000 habitants $/ \mathrm{km}^{2}$ sur les îles urbaines les plus peuplées dont la capitale, Malé). Cette forte pression démographique a constitué au cours des dernières décennies un facteur majeur de l'augmentation de la vulnérabilité, sous l'effet de divers processus : (i) l'accroissement de l'exposition de la population et des systèmes de production à ces aléas, en raison de leur implantation à très faible distance de la mer; (ii) la dégradation, voire la destruction, de zones tampons essentielles, comme les récifs coralliens ou les cordons sableux littoraux, sous l'effet de la réalisation d'aménagements (ports, par exemple), de l'empiètement du bâti sur ces systèmes côtiers sensibles, de la déforestation, de l'extraction de matériaux ou d'opérations de remblayage ; (iii) les effets pervers de la démultiplication des ouvrages de protection qui, bien qu'ils aient souvent 


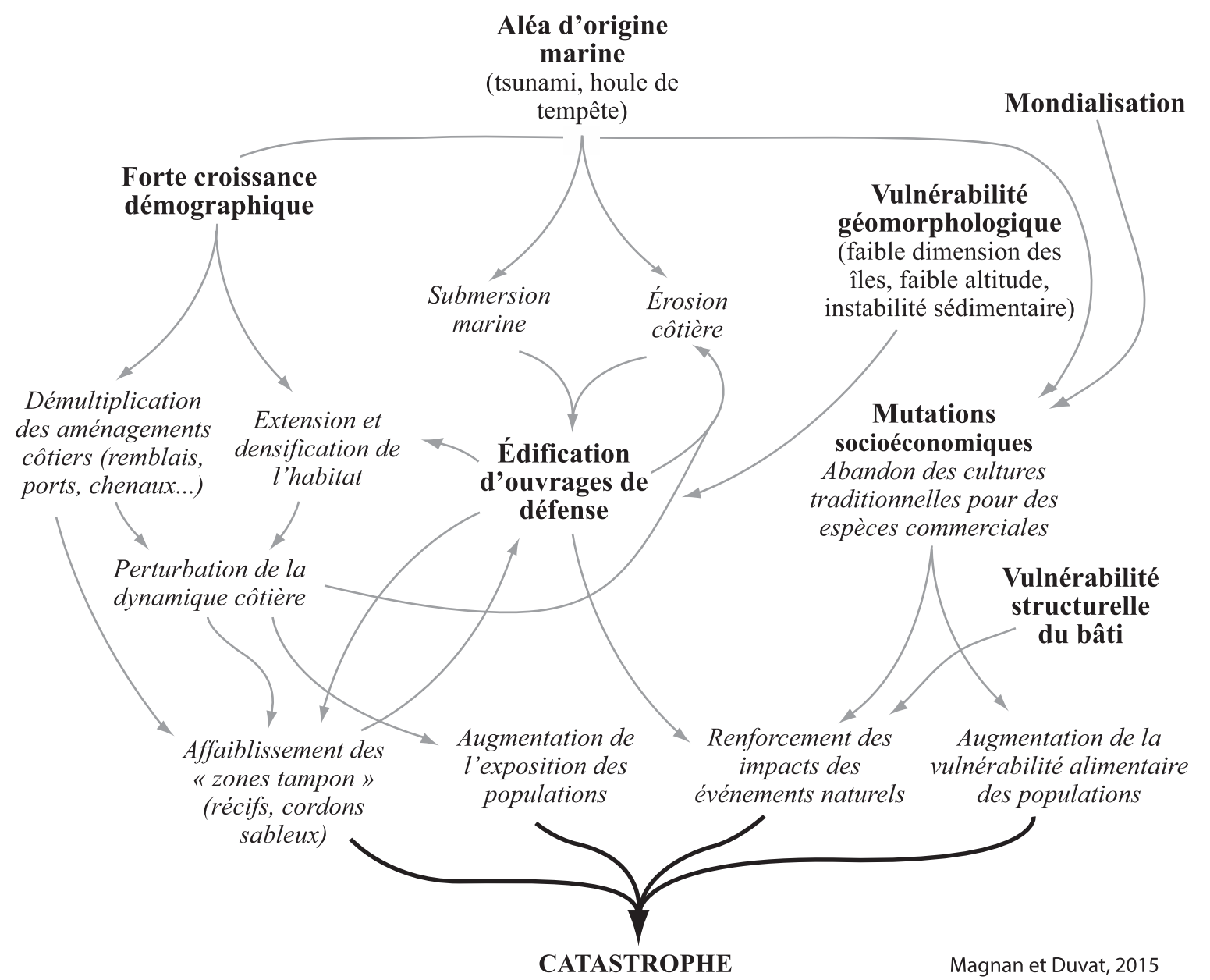

Fig. 3. Le système du risque révélé par le tsunami du 26 décembre 2004 aux Maldives.

déstabilisé les îles coralliennes sur lesquelles ils ont été construits, ont pourtant favorisé la densification et l'extension du bâti (Cazes-Duvat, 2001, 2005, 2007). On retrouve ainsi, dans ce petit État insulaire, les principaux fondements de la vulnérabilité des sociétés tels qu'ils ont été présentés plus haut.

Au-delà de ces premiers facteurs, les impacts du tsunami du 26 décembre 2004 ont révélé des causes plus profondes de la vulnérabilité actuelle de ce territoire (Fig. 3). Environ quatre heures après le séisme de Sumatra (magnitude de 9,3 sur l'échelle de Richter), des vagues de tsunami de 1,2 à 4,2 m de hauteur ont atteint les Maldives, submergeant $57 \%$ de la surface habitée. $35 \%$ des îles habitées ont été balayées par les vagues sur la totalité de leur surface et $29 \%$ sur le tiers de celle-ci. Ce tsunami qui a fait relativement peu de morts (82, auxquels s'ajoutent 26 disparus) par comparaison avec la situation d'autres pays (180 000 morts en Indonésie, au Sri Lanka et en Inde) a cependant eu d'importants effets puisqu'il a engendré la migration immédiate de 20000 à 25000 Maldiviens vers d'autres îles de l'archipel, en raison des dommages causés à l'habitat (8 500 maisons touchées) et aux bâtiments publics (écoles, pharmacies et dispensaires, notamment), et de l'interruption de l'approvisionnement en eau sur de nombreuses îles, sous l'effet de la salinisation de l'eau des puits et de la perte des maigres stocks d'eau pluviale (Magnan, 2006). Ainsi, cet événement a révélé, dans les atolls extérieurs ${ }^{5}$, la forte vulnérabilité structurelle du bâti, qui était alors encore largement constitué de blocs de corail non cimentés ou de matériaux légers, d'origine végétale ou importée (toile). Ce tsunami a également montré la très grande vulnérabilité alimentaire et sanitaire de la population de ces atolls, d'abord en raison de sa forte dépendance vis-à-vis de l'eau de la lentille souterraine. Ce constat a d'ailleurs incité les autorités à équiper, rapidement après cet événement, ces îles en réservoirs d'eau adéquats (plus de 21000 ménages en ont bénéficié dans une centaine d'îles) et en usines de dessalement de l'eau de mer (55 dans 35 îles). En parallèle, la destruction des

5 En dehors de Malé. 
principales cultures (bananes, papayes, fruit à pain) a révélé la vulnérabilité d'une agriculture qui s'est tournée au cours des dernières décennies vers des espèces très sensibles à une salinisation des sols, au détriment d'espèces plus adaptées aux conditions écologiques locales, telles que le cocotier ou le pandanus. Cette évolution des choix agricoles traduit d'ailleurs une perte progressive des liens des Maldiviens avec leur environnement (non-conscience des impacts potentiels de tels changements sur la sécurité alimentaire), que le développement de l'agriculture commerciale accentue. La population des îles fortement investies dans l'agriculture commerciale (environ 30 îles) a perdu, avec la destruction des récoltes, non seulement tout moyen de s'alimenter, mais aussi les revenus lui permettant d'acheter des produits alimentaires. Ces îles ont donc été particulièrement touchées par l'inflation, le tsunami ayant engendré une hausse de $67 \%$ du prix de la noix de coco et de $80 \%$ de celui de la banane. La perte de la flotte de pêche sur $25 \%$ des îles habitées, due à l'absence d'infrastructures portuaires protégeant les embarcations (simple jetée, le plus souvent), a exacerbé ces difficultés alimentaires, les Maldiviens étant fortement dépendants des ressources halieutiques au quotidien (190 kg/hab/an) (Doumenge, 2005). Ce tsunami a ainsi mis en évidence le rôle majeur des évolutions socioéconomiques récentes dans l'augmentation de la vulnérabilité de la population face aux événements marins. Le développement économique des Maldives, s'il a eu des effets bénéfiques dans les domaines de la santé et de l'éducation, par exemple, a aussi par d'autres biais (mutations de l'agriculture et des modes de consommation alimentaire) contribué à accroître la vulnérabilité de la population. Au total, les dommages causés par ce tsunami ont été évalués à $470 \mathrm{M} \$$, représentant $62 \%$ du PNB.

Ce tsunami a aussi révélé des facteurs-clés de résilience, qui ont démontré leur efficacité en contribuant à la rapidité de la reconstruction et au retour à la normale. Il a, en particulier, mis en évidence le rôle de deux facteurs, les ressources économiques extérieures (tourisme et aide internationale) et les solidarités socioterritoriales dans l'absorption des impacts de cet événement. Le tourisme, qui joue un rôle majeur dans l'économie maldivienne $\left(1^{\mathrm{er}}\right.$ secteur d'activité avec $28 \%$ du PNB, $38 \%$ des revenus du gouvernement ${ }^{6}, 75 \%$ des devises et environ 11000 emplois $^{7}$ ), a été fortement affecté par le tsunami, avec la fermeture de 19 îles-hôtels sur 87 (chute immédiate de $25 \%$ de la capacité d'accueil) et un effondrement brutal et massif des arrivées touristiques (baisse de $70 \%$ en janvier 2005 par rapport à janvier 2004). Cette baisse très nette de l'activité a entraîné une diminution

\footnotetext{
6 Source : Government of the Maldives, 2012.

7 Source : Government of the Maldives, 2006 (pas de données officielles plus récentes).
}

de $80 \mathrm{M} \$$ des revenus du gouvernement maldivien en 2005. Mais ce secteur d'activité économique, parce qu'il dépend largement de capitaux extérieurs (compagnies internationales soutenues par leurs assurances), a connu un redressement très rapide, la majorité des îles-hôtels touchées ayant rouvert en 2006. La forte résilience du tourisme a soutenu la résilience plus globale de l'économie maldivienne, à travers les revenus et les devises qu'il procure au gouvernement maldivien et les emplois qu'il fournit à une partie de la population. Ce secteur a depuis lors continué à se développer, le nombre des îles-hôtels et des lits touristiques étant respectivement passé de 87 et 16500 en 2004 à 105 et 22889 en 2012. Ces chiffres démontrent l'importance des investissements extérieurs et de la diversification de l'économie dans la résilience $\mathrm{d}^{\prime}$ un pays en développement, en particulier lorsqu'il est insulaire. L'importance cruciale de l'aide extérieure a été encore plus manifeste par le rôle que la coopération internationale a joué dans la reconstruction (393 M\$), en finançant la réparation et la construction de logements (qui ont capté $30 \%$ de cette aide), le rétablissement de l'accès à l'eau et des transports ( $28 \%$ ), et le soutien au redressement des secteurs de l'agriculture et de la pêche $(6 \%)$.

Le second facteur majeur de la résilience des Maldives face au tsunami de 2004 a été l'existence de fortes solidarités sociales et territoriales pendant et après cet événement. Elles ont pris des formes variées, dont les principales ont été l'accueil de sinistrés, le partage des ressources vitales (eau, nourriture) et le prêt d'argent. Les liens sociaux ont ainsi permis aux 20000 à 25000 Maldiviens qui ont dû abandonner leur habitation de trouver refuge dans leur famille, chez des voisins ou des amis, dans leur île d'origine ou dans une autre île du même atoll pour, dans certains cas, s'y établir durablement. Ces réseaux de solidarité, qui ont joué de manière spontanée un rôle crucial dans l'amortissement des impacts socioéconomiques du tsunami, démontrent une capacité de réponse élevée de la société maldivienne considérée dans son ensemble, en dépit de sa pauvreté.

Comme dans le cas de Katrina, l'exemple maldivien souligne le rôle majeur des modalités d'aménagement $\mathrm{du}$ territoire (plus ou moins productrices de risque), de l'accès aux ressources vitales, et des solidarités sociales et territoriales dans la résonance d'un événement naturel.

\section{Exemple 3 : la tempête Xynthia (février 2010) dans l'Ouest français}

La tempête Xynthia a donné lieu à de nombreuses publications scientifiques dont il ne s'agit pas ici de restituer l'intégralité des contenus. On s'emploiera plutôt à montrer ce que Xynthia a révélé de la vulnérabilité 


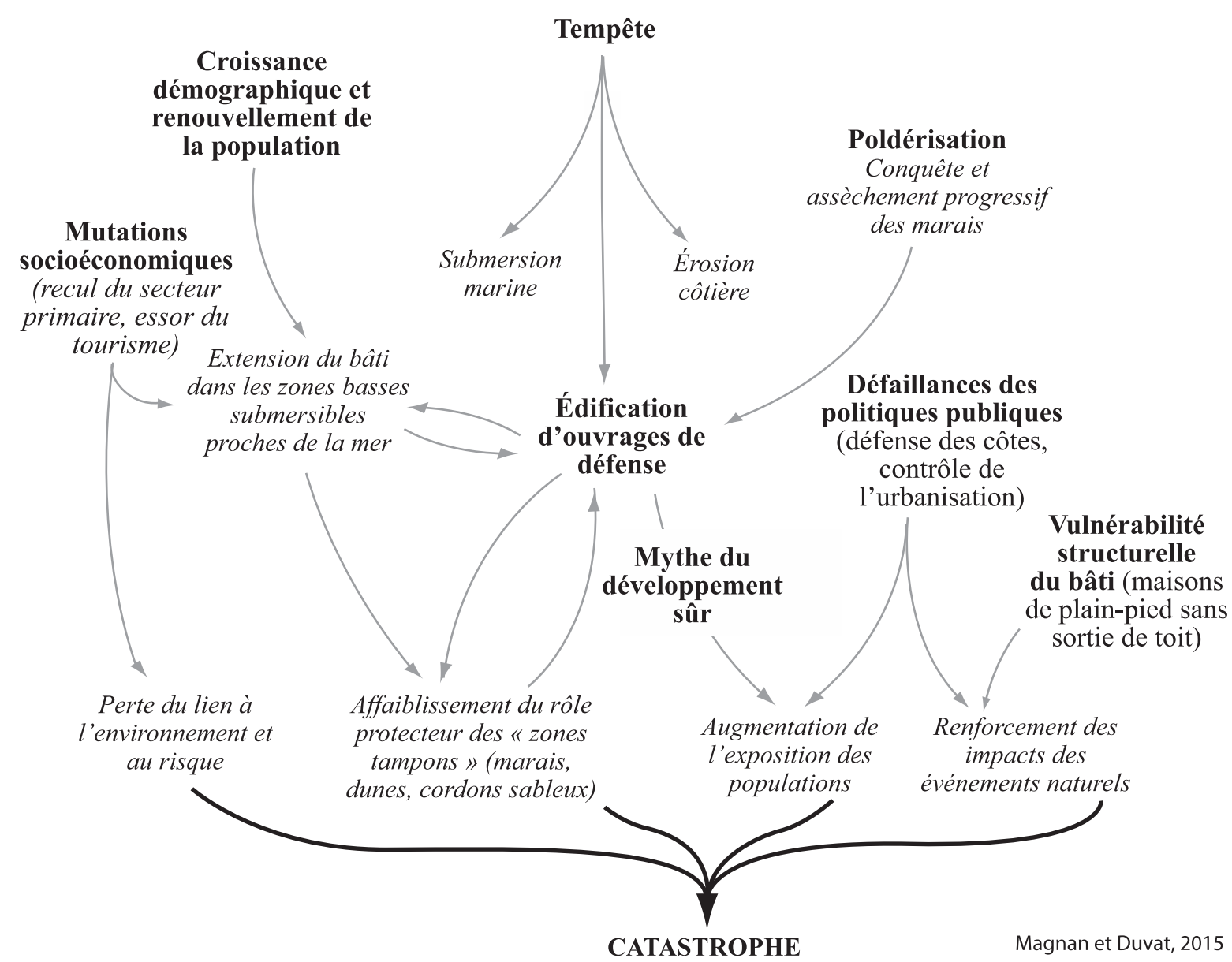

Fig. 4. Le système du risque révélé par la tempête Xynthia (février 2010) en France.

des territoires littoraux de Charente-Maritime et de Vendée (Fig. 4).

$\mathrm{Au}$ moment où Xynthia touche ces deux départements côtiers, la dernière tempête ayant provoqué une submersion rapide remonte à plus d'un demi-siècle (1957). Et pendant ce demi-siècle, sous l'effet d'une forte

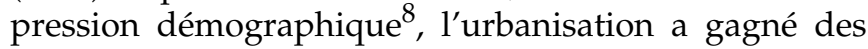
zones basses situées sous le niveau de la mer et constituées de marais maritimes, de dépressions intradunaires et de berges de rivières. Ce faisant, elle a empiété sur des espaces «naturels » amortisseurs d'aléas et porté atteinte à leur rôle de zone tampon entre la mer ou les cours d'eau et les enjeux humains. Ainsi, au sein des polders, de nombreux villages se sont progressivement étendus depuis les points hauts sur lesquels ils avaient été au départ prudemment édifiés vers les zones basses alentour. L'exemple de La Faute-sur-Mer est instructif à cet égard, puisque $70 \%$ des parcelles aménagées à partir

8 Entre 1946 et 2007, la population des 44 communes littorales situées entre le Sud Vendée et La Tremblade a pratiquement doublé, passant de 100000 à 182000 habitants. des années 1980 seront submergées par Xynthia (contre $23 \%$ de celles bâties avant 1950), et près de la moitié de celles bâties entre 2000 et 2010 recouvertes par un mètre d'eau le 28 février 2010 (Vinet et al., 2012). Ces chiffres traduisent bien le fait qu'au fil du temps, ont été urbanisées des zones toujours plus dangereuses.

L'existence de digues côtières et d'ouvrages hydrauliques visant à contrôler le niveau des eaux dans les marais, a, ici comme à la Nouvelle-Orléans, eu pour effet de soutenir le processus d'urbanisation, suivant l'idée que ces ouvrages protégeaient ces terres basses de la submersion et des inondations. Il en est résulté une perte progressive de la conscience du risque au sein de la société (cf. Fig. 1), dont le renouvellement important a évidemment soutenu ce processus ${ }^{9}$. On retrouve ici les mêmes mécanismes de production de la vulnérabilité que ceux qui expliquent la résonance de Katrina à la

\footnotetext{
9 À titre d'exemple, les communes d'Esnandes, de La Fautesur-Mer, de Charron et d'Aytré ont respectivement connu une augmentation de leur population de $179 \%, 87 \%, 70 \%$ et $66 \%$, entre 1962 et 2007.
} 
Nouvelle-Orléans, avec l'occupation progressive par l'homme de zones basses exposées aux aléas, mais considérées comme sûres en raison de la présence d'ouvrages de protection. Ici aussi, donc, le mythe du développement « sûr » a été à l'œuvre.

Pourtant, Xynthia révélera les défaillances de ces ouvrages, qu'il s'agisse des digues côtières (surverse, ouverture de brèches, destruction totale) ou des équipements hydrauliques (écluses, canaux de drainage, etc.), dont beaucoup étaient en mauvais état ou inopérants pendant cet événement. Cette situation tient tout à la fois à la réduction des dépenses publiques affectées à la défense des côtes ${ }^{10}$, à l'abandon des pratiques ancestrales d'entretien de ces ouvrages, et à la dilution des responsabilités en ce domaine, résultant de l'intervention d'acteurs multiples, mais mal identifiés. Ainsi, le rapport de la Cour des comptes de 2012 révèle qu'en CharenteMaritime, les propriétaires et gestionnaires de $95 \%$ du linéaire des digues côtières ne sont pas identifiés, et que les $5 \%$ restants appartiennent à des propriétaires « incapables de les entretenir, faute de moyens et de volonté ». Dans le cas emblématique de La Faute-sur-Mer, ce facteur a joué un rôle important : la digue est, qui borde le cours du Lay et qui a été submergée par Xynthia, devait depuis un certain temps faire l'objet de travaux qui n'avaient toujours pas été réalisés en février 2010, en raison d'un véritable « imbroglio juridique » entre les associations syndicales qui en étaient propriétaires et la commune. En Charente-Maritime, au lendemain de Xynthia, ce sont au total $120 \mathrm{~km}$ de digues (soit environ la moitié du linéaire côtier équipé d'ouvrages de défense) qu'il faut reconstruire.

Les mutations socioéconomiques des dernières décennies ont soutenu l'urbanisation des zones exposées aux aléas submersion et inondation, le recul du secteur primaire ayant favorisé le redéploiement foncier et, avec lui, le développement de stratégies immobilières opportunistes visant à tirer profit de deux processus concomitants, l'augmentation de l'attractivité résidentielle et l'essor du tourisme. Cette urbanisation rapide n'a d'ailleurs rencontré aucun obstacle. Comme l'explique Jean Renard (1983), les premiers plans d'occupation des sols (1967) ont davantage été des « instruments de distribution des droits à construire que des documents d'aménagement ». Par la suite, les difficultés de mise en place des Plans de prévention des risques naturels (PPRN) prévus par la loi Barnier (1995) - qu'un certain nombre de maires ont vus comme des obstacles au développement de leur commune - ont permis la poursuite de l'urbanisation des zones basses.

\footnotetext{
10 Selon la Cour des comptes (2012), les dépenses publiques nécessaires à un entretien correct de ces ouvrages de défense étaient, en Sud-Vendée avant 2010, trois fois inférieures aux besoins réels.
}

Dans le même temps, le remplacement de la population d'agriculteurs et de marins, qui avait une culture de l'environnement, des aléas et du risque, par une population sans culture maritime, et dans certains cas âgée (constituée de 30 à $40 \%$ de retraités, comme à La Fautesur-Mer) et composée de résidents secondaires ( $87 \%$ dans cette même commune), a encore aggravé la situation alors qu'en parallèle, la maison de plain-pied sans sortie de toit tendait à se généraliser (Vinet et al., 2012). L'adoption de ce type d'architecture dans une région exposée à l'aléa submersion traduit bien à son tour la perte de toute conscience du risque.

C'est donc bien ici aussi une combinaison de facteurs alliant la construction en zone exposée, la négligence de l'entretien des systèmes hydrauliques et de défense, la défaillance des politiques publiques et l'augmentation de la vulnérabilité socioéconomique (renouvellement de la population et des formes d'habitat, perte de conscience du risque) qui explique, comme dans le cas de Katrina, la résonance qu'a eue cette tempête.

\section{Conclusion}

Les trois études de cas précédentes démontrent qu'il est possible d'en dégager des éléments communs. Tout se passe finalement comme si différents cas racontaient des histoires différentes dans leurs détails mais qui, dans leurs traits essentiels, présentent des points de convergence. Un tel constat invite à tirer quatre enseignements fondamentaux, qui sont autant de messages-clés : (i) les catastrophes naturelles existent bel et bien ; (ii) la vulnérabilité des sociétés contemporaines s'enracine dans une forme de déni du risque ; (iii) toutes les sociétés, quel que soit leur niveau de développement, sont vulnérables aux aléas naturels ; et (iv) réduire l'impact des catastrophes actuelles constitue une voie pragmatique pour engager un processus d'adaptation au changement climatique.

- Les catastrophes naturelles existent bel et bien. Elles ont ceci de naturel qu'elles sont déclenchées à un moment donné par des aléas naturels, mais ce sont précisément des catastrophes dans la mesure où elles s'enracinent dans des dimensions profondément humaines qui s'expriment à travers des modes de développement déterminés. En définitive, ce sont bien les défaillances de ces modes de développement qui viennent expliquer, lorsqu'un aléa se produit, toute la "chaîne d'impacts " qui s'ensuit. Or, la transformation d'un aléa en catastrophe repose précisément sur l'existence de cette chaîne d'impacts.

- La vulnérabilité des sociétés contemporaines s'enracine dans une forme de déni du risque, et ce déni est lié à au moins trois éléments principaux. D'abord, la perte du lien à l'environnement, qui génère insidieusement et de manière progressive un refus de l'existence 
de contraintes environnementales (en l'occurrence ici, les aléas naturels et leurs impacts physiques). Ensuite, l'oubli des catastrophes passées, de leurs impacts et de leur extension spatiale, qui, combiné à un renouvellement rapide et massif des populations littorales (des «nouveaux arrivants» n'ayant aucune notion des risques dans la zone de destination), conduit en définitive à l'oubli de l'existence même du risque. Enfin, le sentiment de dominer les processus naturels, dans le cadre d'un « mythe du développement sûr ».

- Toutes les sociétés, quel que soit leur niveau de développement, sont vulnérables aux aléas naturels. À l'évidence, des niveaux inégaux de développement créent des formes de vulnérabilité différentes (Magnan, 2013). Cependant, il est illusoire de penser qu'un niveau de développement élevé place les sociétés à l'abri des catastrophes naturelles. De nombreux exemples récents dans des pays développés en témoignent. Cette idée essentielle constitue un message fort dans la perspective du changement climatique et des efforts d'adaptation que ce dernier impose dès maintenant, au Sud comme au Nord. - Réduire l'impact des catastrophes actuelles constitue une voie pragmatique pour engager un processus d'adaptation au changement climatique. Une part importante des enjeux de demain repose en effet sur la résolution des problèmes posés aujourd'hui, en particulier par la réduction des formes d'inadaptation, générées sur le temps long par le processus de (mal)développement (Cardona et al., 2012 ; Noble et al., 2014). Autrement dit, comprendre les processus qui sont à l'origine de la fabrique des catastrophes dans une société donnée ne consiste pas uniquement à pointer des dysfonctionnements, mais aussi et surtout à mettre en lumière les leviers sur lesquels il faut agir (l'aménagement du territoire, la culture du risque, etc.) et un panel de solutions concrètes répondant à la fois aux risques actuels et aux risques futurs. En définitive, cela revient à insister sur le fait que nous savons - et il faut le prendre comme une bonne nouvelle - ce qu'il faut faire pour anticiper, tout simplement parce que nous avons accumulé une grande expérience, à l'échelle de la planète, des aléas naturels et de leurs impacts sur les sociétés littorales. Désormais, l'enjeu véritable consiste donc moins en l'identification de solutions (on en connaît déjà beaucoup) qu'en l'identification des raisons pour lesquelles ces solutions ne sont pas mises en œuvre à l'heure actuelle. Il faut lever les verrous qui contraignent l'action.

Différents cas de catastrophes naturelles nous montrent $\mathrm{qu}^{\prime}$ on peut apprendre du passé (Revet et Langumier, 2013) et qu'il est même indispensable de remonter le temps pour comprendre les «trajectoires de vulnérabilité » (Magnan et al., 2012), autrement dit ces processus par lesquels les sociétés fabriquent les catastrophes, ici naturelles. La raison en est que les grands facteurs de l'augmentation de la vulnérabilité au cours des dernières décennies, s'ils ne sont pas contrecarrés, perdureront. Les identifier avec précision et comprendre le jeu de leurs interactions constituent donc logiquement un préalable indispensable à la mise en œuvre de toute stratégie de réduction pragmatique des risques naturels non seulement actuels, mais aussi futurs. De fait, le changement climatique va en grande partie aggraver par ses impacts des problèmes qui existent déjà et qui sont clairement identifiés. Il s'ensuit que réduire les risques actuels, au-delà de produire des bénéfices immédiats, constitue une voie pragmatique et "sans regret » pour réduire les risques futurs et donc s'adapter au changement climatique. Cela revient à dire que l'incertitude sur les conséquences du changement climatique aux échelles locales ne constitue dès lors pas une barrière infranchissable à la prise de décision et à l'action en faveur de l'adaptation. Analyser les processus de production du risque, comme on l'a fait ici à partir de trois études de cas, doit en effet permettre d'identifier les barrières à lever et les leviers à activer pour anticiper les risques. Cela doit permettre de dresser un bilan objectif des marges de manœuvre dont nous disposons pour orienter la suite de l'histoire.

Les pages précédentes invitent également à voir en la survenue d'une catastrophe naturelle un révélateur des failles de nos modes de développement modernes. Ainsi, parler de «fabrique » des catastrophes revient à se poser la question suivante: nos modèles de développement modernes sont-ils en échec ? C'est une question qui fait grandement sens dans la perspective du changement climatique, tout du moins qui appelle une, voire plusieurs réponses. Dans cette optique, ce que l'on peut retenir de ce texte, c'est qu'il est absolument fondamental de réintroduire l'existence de contraintes environnementales (ici, des aléas naturels) dans nos manières de penser le développement sur le littoral. Nous devons notamment reconnaître que peuvent survenir des aléas auxquels nous sommes mal préparés, de même que nous devons accepter que certains espaces, exposés à de forts aléas, ne sont tout simplement pas aménageables. Une nouvelle philosophie du rapport au risque doit donc émerger, qui passera nécessairement par l'acceptation des contraintes environnementales, en opposition au siècle qui vient de s'écouler et qui a vu l'avènement d'une volonté de s'affranchir systématiquement, et quel qu'en soit le prix, du risque naturel et, au-delà, de la contrainte environnementale.

\section{Références}

Burby, R.J., 2006. Hurricane Katrina and the paradoxes of government disaster policy: bringing about wise governmental decisions for hazardous areas, Annals of the American Academy of Political and Social Science, 604, 1, 171-191. 
Cannon, T., 1994. Vulnerability analysis and the explanation of "natural" disasters, in Varley, A. (Ed.), Disasters, development and environment, Chichester/New York, J. Wiley, 13-30.

Cardona, O.D., van Aalst, M.K., Birkmann, J., Fordham, M., McGregor, G., Perez, R., Pulwarty, R.S., Schipper, E.L.F., Sinh, B.T., 2012. Determinants of risk: exposure and vulnerability, in IPCC, Managing the risks of extreme events and disasters to advance climate change adaptation. Special Report of Working Groups I and II of the Intergovernmental Panel on Climate Change [Field, C.B., Barros, V., Stocker, T.F., Qin, D., Dokken, D.J., Ebi, K.L., Mastrandrea, M.D., Mach, K.J., Plattner, G.-K., Allen, S.K., Tignor, M., Midgley, P.M. (Eds)], Cambridge (UK)/New York, Cambridge University Press, 65-108.

Cazes-Duvat, V., 2001. Le poids des contraintes physiques dans le développement des atolls : l'exemple de l'archipel des Maldives, Les Cahiers d'Outre-Mer, 54, 213, 3-26.

Cazes-Duvat, V., 2005. Les archipels de l'ouest de l'océan Indien face à l'érosion côtière, Annales de géographie, 114, 644, 342-361.

Cazes-Duvat, V., 2007. Les littoraux coralliens des petites îles de l'océan Indien (Mascareignes, Seychelles, Maldives), $2^{\mathrm{e}}$ partie, Aménagement et gestion, Océanis, 31-3/4, 189-334.

Chauveau, É., Chadenas, C., Comentale, B., Pottier, P., Blanlœil, A., Feuillet, T., Mercier, D., Pourinet, L., Rollo, N., Tillier, I., Trouillet, B., 2011. Xynthia: les leçons d'une catastrophe, Cybergeo : European Journal of Geography, 538, http:/ / cybergeo.revues.org/23763

Cour des comptes, 2012. Les enseignements des inondations de 2010 sur le littoral atlantique (Xynthia) et dans le Var. Rapport public thématique.

Cutter, S., 2006. The geography of social vulnerability: race, class and catastrophe, in Understanding Katrina: perspectives from the social sciences, Brooklyn (NY), Social Science Research Council , http://understandingkatrina.ssrc.org/Cutter/.

Doumenge, F., 2005. L'halieutique maldivienne, une ethnoculture millénaire, Archipels, 70, 67-138.

Duvat, V., Magnan, A., 2014. Des catastrophes... «naturelles »?, Paris, Le Pommier.

Duvat, V., Magnan, A., Pouget, F., 2013. Exposure of atoll population to coastal erosion and flooding: a South Tarawa assessment, Kiribati, Sustainability Science, 8, 3, 423-440.

Dworkin, J., 1974. Global trends in natural disasters 1947-1973, Natural Hazard Research, Working paper 26, University of Colorado.

Funabashi, H., 2012. Why the Fukushima nuclear disaster is a man-made calamity, International Journal of Japanese Sociology, 21, 1, 65-75.

Funabashi, Y., Kitazawa, K., 2012. Fukushima in review: a complex disaster, a disastrous response, Bulletin of the Atomic Scientists, 68, 2, 9-21.

Gemenne, F., 2010. What's in a name: social vulnerabilities and the refugee controversy in the wake of Hurricane Katrina, in Afifi, T., Jäger, J. (Eds), Environment, forced migration and social vulnerability, Berlin, Springer.

Guha-Sapir, D., Hergitt, D., Hoyois, P., 2004. Thirty years of natural disasters 1974-2003: the numbers, Louvain-la-Neuve, Presses universitaires de Louvain.
Hasegawa, K., 2012. Facing nuclear risks: lessons from the Fukushima nuclear disaster, International Journal of Japanese Sociology, 21, 1, 84-91.

Kates, R.W., Colten, C.E., Laska, S., Leatherman, S.P., 2006. Reconstruction of New Orleans after Hurricane Katrina: a research perspective, Proceedings of the National Academy of Sciences of the United States of America, 103, 40, 14653-14660.

Laska, S., 2004. What if Hurricane Ivan had not missed New Orleans?, Sociological Inquiry, 78, 2, 174-178.

Magnan, A., 2006. L'évacuation des populations aux Maldives après le tsunami du 26 décembre 2004, Mappemonde, 84, 4, http:/ / mappemonde.mgm.fr/num12/articles / art06403.html.

Magnan, A., 2013. Changement climatique: tous vulnérables? Repenser les inégalités, Paris, Éditions Rue d'Ulm.

Magnan, A., Duvat, V., Garnier, E., 2012. Reconstituer les " trajectoires de vulnérabilité » pour penser différemment l'adaptation au changement climatique, Natures Sciences Sociétés, 20, 1, 82-91.

Mancebo, F., 2006. Katrina et la Nouvelle-Orléans : entre risque « naturel » et aménagement par l'absurde, Cybergéo : European Journal of Geography, 353, http:/ / cybergeo.revues.org/90.

Noble, I.R., Huq, S., Anokhin, Y.A., Carmin, J., Goudou, D., Lansigan, F.P., Osman-Elasha, B., Villamizar, A., 2014. Adaptation needs and options, in IPCC, Climate change 2014: impacts, adaptation, and vulnerability. Part A: global and sectoral aspects. Contribution of Working Group II to the Fifth Assessment Report of the Intergovernmental Panel on Climate Change [Field, C.B., Barros, V., Dokken, D.J., Mach, K.J., Mastrandrea, M.D., Bilir, T.E., Chatterjee, M., Ebi, K.L., Estrada, Y.O., Genova, R.C., Girma, B., Kissel, E.S., Levy, A.N., MacCracken, S., Mastrandrea, P.R., White, L.L. (Eds)], Cambridge (UK)/New York, Cambridge University Press, 833-868.

Nöggerath, J., Geller, R.J., Gusiakov, V.K., 2011. Fukushima: the myth of safety, the reality of geoscience, Bulletin of the Atomic Scientists, 67, 5, 37-46.

Paskoff, R., 2004. Côtes en danger. Paris, L'Harmattan.

Renard, J., 1983. Conflits et consommations de l'espace par le tourisme sur le littoral vendéen, in Groupe SERS-Façade atlantique (Groupe de recherche sur les structures économiques et les rapports sociaux), Les faits d'occupation conflictuelle du littoral. Troisième rapport de recherche, Nantes, Université de Nantes, 168-185.

Revet, S., Langumier, J., 2013. Le gouvernement des catastrophes, Paris, Karthala.

Vinet, F., Defossey, S., Rey, T., Boissier, L., 2012. Le processus de production du risque "submersion marine» en zone littorale : l'exemple des territoires "Xynthia », Norois, 222, http://norois.revues.org/3834.

WEF (World Economic Forum), 2014. Global Risks 2014: ninth edition. Insight report, World Economic Forum, http:// reports.weforum.org/global-risks-2014.

Wisner, B., Blaikie, P., Cannon, T., Davis, I., 2004. At risk. Natural hazards, people's vulnerability and disasters, London/ New York, Routledge. 